\title{
Correction to: Genomic Subtyping in Bladder Cancer
}

\section{Tuomas Jalanko $^{1,2}$ (D) $\cdot$ Joep J. de Jong ${ }^{3} \cdot$ Ewan A. Gibb $^{4} \cdot$ Roland Seiler $^{5}$ (D) $\cdot$ Peter C. Black $^{1}$ (D)}

Published online: 14 May 2020

(C) Springer Science+Business Media, LLC, part of Springer Nature 2020

\section{Correction to: Current Urology Reports (2020) 21(2). https://doi.org/10.1007/s11934-020-0960-y}

The original version of this article contained a mistake. The included Conflict of Interest statement was incorrect.

The correct Conflict of Interest statement is:

Conflict of Interest Tuomas Jalanko, Joep J. de Jong, Ewan A. Gibb and Roland Seiler each declare no potential conflicts of interest. Peter C. Black has a patent on genomic markers in bladder cancer with Decipher Biosciences, Vancouver, BC, Canada.

Publisher's Note Springer Nature remains neutral with regard to jurisdictional claims in published maps and institutional affiliations.

The online version of the original article can be found at https://doi.org/ 10.1007/s11934-020-0960-y

Peter C. Black

pblack@mail.ubc.ca

Tuomas Jalanko

tuomas.jalanko@hus.fi

Joep J. de Jong

j.j.dejong@erasmusmc.nl

Ewan A. Gibb

ewan.gibb@decipherbio.com

Roland Seiler

r_seiler@gmx.ch; roland.seiler@insel.ch
Vancouver Prostate Centre, Department of Urologic Sciences, University of British Columbia, Vancouver, Canada

2 Department of Urology, Helsinki University, Helsinki, Finland

3 Department of Urology, Erasmus MC Cancer Institute, Rotterdam, The Netherlands

4 Decipher Biosciences, Vancouver, Canada

5 Department of Urology, University of Bern, Bern, Switzerland 